ESAIM: COCV 19 (2013) 740-753

DOI: $10.1051 / \mathrm{cocv} / 2012031$
ESAIM: Control, Optimisation and Calculus of Variations

www.esaim-cocv.org

\title{
GAMMA-CONVERGENCE RESULTS FOR PHASE-FIELD APPROXIMATIONS OF THE 2D-EULER ELASTICA FUNCTIONAL
}

\author{
LuCA Mugnai
}

\begin{abstract}
We establish some new results about the $\Gamma$-limit, with respect to the $L^{1}$-topology, of two different (but related) phase-field approximations $\left\{\mathcal{E}_{\varepsilon}\right\}_{\varepsilon},\left\{\widetilde{\mathcal{E}}_{\varepsilon}\right\}_{\varepsilon}$ of the so-called Euler's Elastica Bending Energy for curves in the plane. In particular we characterize the $\Gamma$-limit as $\varepsilon \rightarrow 0$ of $\mathcal{E}_{\varepsilon}$, and show that in general the $\Gamma$-limits of $\mathcal{E}_{\varepsilon}$ and $\widetilde{\mathcal{E}}_{\varepsilon}$ do not coincide on indicator functions of sets with non-smooth boundary. More precisely we show that the domain of the $\Gamma$-limit of $\widetilde{\mathcal{E}}_{\varepsilon}$ strictly contains the domain of the $\Gamma$-limit of $\mathcal{E}_{\varepsilon}$.
\end{abstract}

Mathematics Subject Classification. 49J45, 34K26, 49Q15, 49Q20.

Received November 25, 2010. Revised January 26, 2012.

Published online June 3, 2013.

\section{INTRODUCTION}

In this paper we present some new results about the sharp interface limit of two families of phase-field functionals involving the so-called Cahn-Hilliard energy functional and its $L^{2}$ - gradient. To introduce the two families of functionals we are going to study let us recall that the Cahn-Hilliard energy $\left\{\mathcal{P}_{\varepsilon}\right\}_{\varepsilon}$ is defined as follows: given $\Omega \subset \mathbb{R}^{d}$ open, bounded and with smooth boundary we set

$$
\mathcal{P}_{\varepsilon}(u):= \begin{cases}\int_{\Omega} \frac{\varepsilon}{2}|\nabla u|^{2}+\frac{W(u)}{\varepsilon} \mathrm{d} x & \text { if } u \in W^{1,2}(\Omega), \\ +\infty & \text { otherwise on } L^{1}(\Omega),\end{cases}
$$

where $\varepsilon>0$ is a parameter representing the typical "diffuse interface width", and $W \in C^{3}\left(\mathbb{R}, \mathbb{R}^{+} \cup\{0\}\right)$ is a double-well potential with two equal minima (throughout the paper we make the choice $W(s):=\left(1-s^{2}\right)^{2} / 4$, though most of the results we obtain hold true for a wider class of potentials). The families of functionals $\left\{\widetilde{\mathcal{E}}_{\varepsilon}\right\}_{\varepsilon}$, $\left\{\mathcal{E}_{\varepsilon}\right\}_{\varepsilon}$ we consider in this paper are respectively defined by

$$
\begin{gathered}
\widetilde{\mathcal{E}}_{\varepsilon}:=\left(\mathcal{P}_{\varepsilon}+\mathcal{W}_{\varepsilon}\right): L^{1}(\Omega) \rightarrow[0,+\infty], \\
\text { where } \mathcal{W}_{\varepsilon}(u):= \begin{cases}\frac{1}{\varepsilon} \int_{\Omega}\left(\varepsilon \Delta u-\frac{W^{\prime}(u)}{\varepsilon}\right)^{2} \mathrm{~d} x & \text { if } u \in C^{2}(\Omega), \\
+\infty & \text { elsewhere on } L^{1}(\Omega),\end{cases}
\end{gathered}
$$

Keywords and phrases. $\Gamma$-convergence, relaxation, singular perturbation, geometric measure theory.

1 Luca Mugnai, Max Planck Institute for Mathematics in the Sciences, Inselstr. 22, 04103 Leipzig, Germany.

mugnai@mis.mpg.de 
and

$$
\begin{gathered}
\mathcal{E}_{\varepsilon}:=\left(\mathcal{P}_{\varepsilon}+\mathcal{B}_{\varepsilon}\right): L^{1}(\Omega) \rightarrow[0,+\infty], \\
\text { where } \mathcal{B}_{\varepsilon}(u):= \begin{cases}\frac{1}{\varepsilon} \int_{\Omega}\left|\varepsilon \nabla^{2} u-\frac{W^{\prime}(u)}{\varepsilon} \nu_{u} \otimes \nu_{u}\right|^{2} \mathrm{~d} x & \text { if } u \in C^{2}(\Omega), \\
+\infty & \text { elsewhere on } L^{1}(\Omega),\end{cases}
\end{gathered}
$$

and $\nu_{u}$ is a unit vector-field such that

$$
\nu_{u}=\frac{\nabla u}{|\nabla u|} \text { on }\{\nabla u \neq 0\} \text { and } \nu_{u} \equiv \text { const. on }\{\nabla u=0\} \text {. }
$$

We remark that $\mathcal{W}_{\varepsilon}(u)$ represents the (rescaled) norm of the $L^{2}$-gradient of $\mathcal{P}_{\varepsilon}$ at $u$, and that $\mathcal{W}_{\varepsilon}$ and $\mathcal{B}_{\varepsilon}$ are linked by the relation

$$
\operatorname{tr}\left[\varepsilon \nabla^{2} u-\frac{W^{\prime}(u)}{\varepsilon} \nu_{u} \otimes \nu_{u}\right]=\varepsilon \Delta u-\frac{W^{\prime}(u)}{\varepsilon}
$$

Hence we have

$$
d\left|\varepsilon \nabla^{2} u-\frac{W^{\prime}(u)}{\varepsilon} \nu_{u} \otimes \nu_{u}\right|^{2} \geq\left(\varepsilon \Delta u_{\varepsilon}-\frac{W^{\prime}\left(u_{\varepsilon}\right)}{\varepsilon}\right)^{2} .
$$

Next, we briefly summarize the known results about the sharp interface limit of $\left\{\widetilde{\mathcal{E}}_{\varepsilon}\right\}_{\varepsilon}$ and $\left\{\mathcal{E}_{\varepsilon}\right\}_{\varepsilon}$. The starting point for the analysis of the asymptotic behavior, as $\varepsilon \rightarrow 0$, of $\left\{\widetilde{\mathcal{E}}_{\varepsilon}\right\}_{\varepsilon}$ and $\left\{\mathcal{E}_{\varepsilon}\right\}_{\varepsilon}$ is a well-known result, due to Modica and Mortola, establishing the $\Gamma$-convergence of $\mathcal{P}_{\varepsilon}$ to the area functional. More precisely, in [16], it has been proved that the $\Gamma\left(L^{1}(\Omega)\right)$-limit of the family $\left\{\mathcal{P}_{\varepsilon}\right\}_{\varepsilon}$ is given by

$$
\Gamma\left(L^{1}(\Omega)\right)-\lim _{\varepsilon \rightarrow 0} \mathcal{P}_{\varepsilon}(u)=\mathcal{P}(u):= \begin{cases}\frac{c_{0}}{2} \int_{\Omega} \mathrm{d}|\nabla u| & \text { if } u \in B V(\Omega,\{-1,1\}), \\ +\infty & \text { elsewhere in } L^{1}(\Omega),\end{cases}
$$

where $c_{0}:=\int_{-1}^{1} \sqrt{2 W(s)} \mathrm{d} s$. We remark that for every $u \in B V(\Omega,\{-1,1\})$ we can write $u=2 \chi_{E}-1=$ : $\mathbb{1}_{E}$, where $\chi_{E}$ denotes the characteristic function of the finite perimeter set $E:=\{u \geq 1\}$. Hence $\mathcal{P}(u)=$ $c_{0} \mathcal{H}^{d-1}\left(\partial^{*} E\right)$ where $\mathcal{H}^{d-1}$ denotes the $(d-1)$-dimensional Hausdorff measure in $\mathbb{R}^{d}$ and $\partial^{*} E$ denotes the reduced boundary of $E$ (see [19]).

The main result concerning the $\Gamma$-convergence of $\left\{\widetilde{\mathcal{E}}_{\varepsilon}\right\}_{\varepsilon}$ has been established, for $d=2$ and $d=3$, by Röger and Schätzle in [18] and independently, but only in the case $d=2$, by Tonegawa and Yuko in [17], partially answering to a conjecture of De Giorgi (see [9]). In particular in [18] the authors proved that for $d=2$ or 3 and $E \subset \Omega$ open and with $C^{2}$-smooth boundary, we have

$$
\Gamma\left(L^{1}(\Omega)\right)-\lim _{\varepsilon \rightarrow 0} \widetilde{\mathcal{E}}_{\varepsilon}\left(\mathbb{1}_{E}\right)=c_{0} \int_{\Omega \cap \partial E}\left[1+\left|\mathbf{H}_{\partial E}(x)\right|^{2}\right] \mathrm{d} \mathcal{H}^{d-1}(x),
$$

where $\mathbf{H}_{\partial E}(x)$ denotes the mean curvature vector of $\partial E$ in the point $x \in \partial E$. When $d=2$ we call the functional on the right hand side of (1.7) the Euler's Elastica Functional.

The sequence of functionals $\left\{\mathcal{E}_{\varepsilon}\right\}_{\varepsilon}$ has been introduced in [3] in connection with the problem of finding a diffuse interface approximation of the Gaussian curvature. As a straightforward consequence of the results established in [3] it follows that, again for $d=2,3$ and $E \subset \Omega$ open with $C^{2}$-smooth boundary, we have

$$
\Gamma\left(L^{1}(\Omega)\right)-\lim _{\varepsilon \rightarrow 0} \mathcal{E}_{\varepsilon}\left(\mathbb{1}_{E}\right)=c_{0} \int_{\Omega \cap \partial E}\left[1+\left|\mathbf{B}_{\partial E}(x)\right|^{2}\right] \mathrm{d} \mathcal{H}^{d-1}(x),
$$

where this time $\mathbf{B}_{\partial E}(x)$ denotes the second fundamental form of $\partial E$ in the point $x \in \partial E$. 
In the present paper we restrict to the case $d=2$, and investigate the behavior of $\left\{\widetilde{\mathcal{E}}_{\varepsilon}\right\}_{\varepsilon}$ and $\left\{\mathcal{E}_{\varepsilon}\right\}_{\varepsilon}$ along sequences $\left\{u_{\varepsilon}\right\}_{\varepsilon} \subset C^{2}(\Omega)$ such that

$$
\lim _{\varepsilon \rightarrow 0}\left\|u_{\varepsilon}-\mathbb{1}_{E}\right\|_{L^{1}(\Omega)}=0, \quad \mathbb{1}_{E} \in B V(\Omega,\{-1,1\}),
$$

removing the $C^{2}$-regularity assumption on the boundary of the limit set $E$. In other words we aim at proving a full $\Gamma$-convergence result, on the whole space $L^{1}(\Omega)$.

We recall that if a family of functionals $\Gamma$-converges, and a certain equicoercivity property holds, then the minimizers of such family converge to the minimizers of the $\Gamma$ - limit. Therefore, though the proofs of our main results are relatively easy and short, we expect that a description of the $\Gamma$ - limit, besides its possible mathematical interest, may be of some relevance at least for those applications, such as $[5,10-12,15]$, where the families $\left\{\widetilde{\mathcal{E}}_{\varepsilon}\right\}_{\varepsilon}$ and $\left\{\mathcal{E}_{\varepsilon}\right\}_{\varepsilon}$ are introduced to formulate, and solve numerically, a "diffuse interface" variational problem whose solutions are expected to converge, as $\varepsilon \rightarrow 0$, to the solutions of a given sharp interface minimum problem.

In synthesis our results are the following: we identify the $\Gamma\left(L^{1}(\Omega)\right)-\lim _{\varepsilon \rightarrow 0} \mathcal{E}_{\varepsilon}$, and we show the existence of functions $\mathbb{1}_{E} \in B V(\Omega,\{-1,1\})$ such that

$$
\Gamma\left(L^{1}(\Omega)\right)-\lim _{\varepsilon \rightarrow 0} \widetilde{\mathcal{E}}_{\varepsilon}\left(\mathbb{1}_{E}\right)<\Gamma\left(L^{1}(\Omega)\right)-\lim _{\varepsilon \rightarrow 0} \mathcal{E}_{\varepsilon}\left(\mathbb{1}_{E}\right) .
$$

Hence, the sharp interface limits of $\left\{\widetilde{\mathcal{E}}_{\varepsilon}\right\}_{\varepsilon}$ and $\left\{\mathcal{E}_{\varepsilon}\right\}_{\varepsilon}$ in general do not coincide, although in two space dimensions, by (1.7) and (1.8) and

$$
\left|\mathbf{B}_{\partial E}(x)\right|^{2}=\left|\mathbf{H}_{\partial E}(x)\right|^{2}
$$

we have

$$
\Gamma\left(L^{1}(\Omega)\right)-\lim _{\varepsilon \rightarrow 0} \mathcal{E}_{\varepsilon}\left(\mathbb{1}_{E}\right)=\Gamma\left(L^{1}(\Omega)\right)-\lim _{\varepsilon \rightarrow 0} \widetilde{\mathcal{E}}_{\varepsilon}\left(\mathbb{1}_{E}\right)
$$

for every $E \subset \Omega$ open and with smooth boundary.

In order to better explain our results, we remark that, since $\Gamma$-limits are necessarily lower semi-continuous functionals (see [7], Prop. 4.16), in view of (1.7)-(1.10), a candidate for the $\Gamma$-limit of both $\widetilde{\mathcal{E}}_{\varepsilon}$ and $\mathcal{E}_{\varepsilon}$ is the lower semi-continuous envelope (with respect to the $L^{1}(\Omega)$-topology) of the functional

$$
\mathcal{F}(u):= \begin{cases}\int_{\Omega \cap \partial E}\left[1+\left|\mathbf{H}_{\partial E}\right|^{2}\right] \mathrm{d} \mathcal{H}^{1} & \text { if } u=\mathbb{1}_{E} \text { and } \Omega \cap \partial E \in C^{2}, \\ +\infty & \text { otherwise on } L^{1}(\Omega),\end{cases}
$$

that is the functional

$$
\begin{aligned}
\overline{\mathcal{F}}(u) & :=\inf \left\{\liminf _{k \rightarrow \infty} \mathcal{F}\left(u_{k}\right): L^{1}(\Omega)-\lim _{k \rightarrow \infty} u_{k}=u\right\} \\
& =\sup \left\{\mathcal{G}(u): \mathcal{G} \leq \mathcal{F} \text { on } L^{1}(\Omega), \mathcal{G} \text { is lower semi-continuous on } L^{1}(\Omega)\right\} .
\end{aligned}
$$

Since by [1], Theorem 3.2, we have $\overline{\mathcal{F}}\left(\mathbb{1}_{E}\right)=\mathcal{F}\left(\mathbb{1}_{E}\right)$ whenever $\Omega \cap \partial E$ is of class $W^{2,2}$, by (1.7), (1.8) and the definition of $\overline{\mathcal{F}}$, we can conclude that

$$
\Gamma\left(L^{1}(\Omega)\right)-\lim _{\varepsilon \rightarrow 0} \widetilde{\mathcal{E}}_{\varepsilon} \leq c_{0} \overline{\mathcal{F}} \text { on } L^{1}(\Omega), \quad \Gamma\left(L^{1}(\Omega)\right)-\lim _{\varepsilon \rightarrow 0} \mathcal{E}_{\varepsilon} \leq c_{0} \overline{\mathcal{F}} \text { on } L^{1}(\Omega) .
$$

We can now rephrase the results we obtain as follows: we prove that $\Gamma\left(L^{1}(\Omega)\right)-\lim _{\varepsilon \rightarrow 0} \mathcal{E}_{\varepsilon}=c_{0} \overline{\mathcal{F}}$, and we show that there exist $\mathbb{1}_{E} \in B V(\Omega,\{-1,1\})$ such that $\Gamma\left(L^{1}(\Omega)\right)-\lim _{\varepsilon \rightarrow 0} \widetilde{\mathcal{E}}_{\varepsilon}\left(\mathbb{1}_{E}\right)<c_{0} \overline{\mathcal{F}}\left(\mathbb{1}_{E}\right)=+\infty$. More precisely: In Theorem 4.1, we show that the assumption $\sup _{\varepsilon>0} \mathcal{E}_{\varepsilon}\left(u_{\varepsilon}\right)<+\infty$, implies additional "regularity" on the support of the measure $\mu:=\theta \mathcal{H}_{L_{M}}^{1}$ arising as limit of the energy density measures

$$
\mu_{\varepsilon}:=\left[\varepsilon / 2\left|\nabla u_{\varepsilon}\right|^{2}+W\left(u_{\varepsilon}\right) / \varepsilon\right] \mathcal{L}_{\llcorner}^{d} \Omega
$$


(here $\mathcal{L}_{L}^{d}$ denotes the Lebesgue measure on $\mathbb{R}^{d}$ restricted to $\Omega$ ). Namely we establish that in every point of $M \cap \Omega$ a (unique) tangent-line to $M$ is well defined. Hence, in Corollary 4.4, by means of a characterization of $\overline{\mathcal{F}}$ obtained in [2] (see also Prop. 2.5) we show that $\Gamma\left(L^{1}(\Omega)\right)-\lim _{\varepsilon \rightarrow 0} \mathcal{E}_{\varepsilon}=c_{0} \overline{\mathcal{F}}$.

For what concerns the family $\left\{\widetilde{\mathcal{E}}_{\varepsilon}\right\}_{\varepsilon}$, in Corollary 4.5 we show that in general the support of the limit measure does not necessarily have an unique tangent line in every point. This difference in regularity between the support of the two limit measures is related to the existence of so called "saddle shaped solutions" to the semilinear elliptic equation $-\Delta U+W^{\prime}(U)=0$ on $\mathbb{R}^{2}$ (see $[6,8]$ ). In particular we obtain the existence of $\mathbb{1}_{E} \in B V(\Omega,\{-1,1\})$ such that

$$
\Gamma\left(L^{1}(\Omega)\right)-\lim _{\varepsilon \rightarrow 0} \widetilde{\mathcal{E}}_{\varepsilon}\left(\mathbb{1}_{E}\right)<c_{0} \overline{\mathcal{F}}\left(\mathbb{1}_{E}\right)=\Gamma\left(L^{1}(\Omega)\right)-\lim _{\varepsilon \rightarrow 0} \mathcal{E}_{\varepsilon}\left(\mathbb{1}_{E}\right)=+\infty .
$$

We remark that we do not expect an analogue of Theorem 4.1 to hold in space dimensions $d>2$. In fact, to prove Theorem 4.1 we make use of some regularity results obtained in [13], that are valid only for generalized ( $d-1$ )-dimensional hypersurfaces (namely curvature varifolds, see Def. 2.2) with (generalized) second fundamental form in $L^{p}$ for some $p>(d-1)$. Moreover, though we expect that an analogue of Corollary 4.4 holds also (at least) when $d=3$, to prove such a result we would probably need a different approach. In fact, in the proof of Corollary 4.4 we make an essential use of an "explicit" representation of $\overline{\mathcal{F}}$, that has been established in [2] and is available only in two space dimensions.

The paper is organized as follows. In Section 2 we fix some notation, and recall some results about varifolds and the lower semi-continuous envelope of $\mathcal{F}$. In Section 3, for the readers convenience, we briefly recall some of the main results of $[3,17,18]$. In Section 4 we state and prove our main results, namely Theorem 4.1, Corollary 4.4 and Corollary 4.5 .

\section{Notation AND PRELIMinary RESUlts}

\subsection{General notation}

Throughout the paper we adopt the following notation. By $\Omega$ we denote an open bounded connected subset of $\mathbb{R}^{2}$ with smooth boundary. By $B_{R}(x):=\left\{z \in \mathbb{R}^{2}:|z|<R\right\}$ we denote the euclidean open ball of radius $R$ centered in $x$.

By $\mathcal{L}^{2}$ we denote the 2-dimensional Lebesgue-measure, and by $\mathcal{H}^{1}$ the one-dimensional Hausdorff measure.

For every set $E \subseteq \mathbb{R}^{2}$ we denote by $\mathbb{1}_{E}$ the function such that $\mathbb{1}_{E}(x)=1$ if $x \in E, \mathbb{1}_{E}(x)=-1$ if $x \notin E$. We denote by $\bar{E}$ and $\partial E$ respectively the closure and the topological boundary of $E$.

We say that $E \subset \Omega$ is of class $W^{2,2}$ (resp. $C^{k}, k \geq 1$ ) in $\Omega$, and write $E \in \mathscr{W}^{2,2}(\Omega)$ (resp. $E \in \mathscr{C}^{k}(\Omega)$ ) if $E$ is open in $\Omega$ and, locally near every $x \in \partial E \cap \Omega$, the set $E$ can be represented (up to rigid motions) as the subgraph of a function of class $W^{2,2}$ (resp. $C^{k}$ ).

We say that a set $E \subset \mathbb{R}^{2}$ has finite perimeter in $\Omega$ if $\mathbb{1}_{E} \in B V(\Omega)$. Moreover if $E$ has finite perimeter by $\partial^{*} E$ we denote its reduced boundary (see [19]).

We endow the space of the $(2 \times 2)$ matrices $M=\left(m_{i j}\right) \in \mathbb{R}^{2 \times 2}$ (resp. $2^{3}$ tensors $\left.T=\left(t_{i j k}\right) \in \mathbb{R}^{2^{3}}\right)$ with the norm

$$
|M|^{2}:=\operatorname{tr}\left(M^{T} M\right)=\sum_{i, j=1}^{2}\left(m_{i j}\right)^{2} \quad\left(\text { resp. }|T|^{2}:=\sum_{i, j, k=1}^{2}\left(t_{i j k}\right)^{2}\right),
$$

where $M^{T}$ is the transposed of $M$.

Let $u \in C^{2}(\Omega)$, we define

$$
\nu_{u}:=\frac{\nabla u}{|\nabla u|}, \quad P^{u}:=\mathrm{Id}-\nu_{u} \otimes \nu_{u}, \quad \text { on }\{\nabla u \neq 0\},
$$


and $\nu_{u}:=\mathbf{e}_{2}, P^{u}:=\operatorname{Id}-\mathbf{e}_{2} \otimes \mathbf{e}_{2}$ on $\{\nabla u=0\}$. Moreover we define the second fundamental form of the ensemble of the level sets of $u$ by

$$
\mathbf{B}_{u}=\left(\frac{\left(P^{u}\right)^{T} \nabla^{2} u P^{u}}{|\nabla u|}\right) \otimes \nu_{u}
$$

on $\{\nabla u \neq 0\}$ and $\mathbf{B}_{u}:=\otimes^{3} \mathbf{e}_{2}$ on $\{\nabla u=0\}$. Similarly we define

$$
A_{i j k}^{u}:=-\sum_{l=1}^{2} P_{i l}^{u}\left[\partial_{l}\left(\left(\nu_{u}\right)_{j}\left(\nu_{u}\right)_{k}\right)\right], \quad(i, j, k \in\{1,2\})
$$

on $\{\nabla u \neq 0\}$ and $A^{u}:=\otimes^{3} \mathbf{e}_{2}$ on $\{\nabla u=0\}$.

\subsection{Geometric measure theory: varifolds}

Let us recall some basic fact in the theory of varifolds, the main bibliographic sources being $[14,19]$.

By $G_{1,2}$ we denote the Grasmannian of 1 -subspaces of $\mathbb{R}^{2}$. We identify $T \in G_{1,2}$ with the projection matrix $P_{T} \in \mathbb{R}^{2 \times 2}$ on $T$, and endow $G_{1,2}$ with the relative distance as a compact subset of $\mathbb{R}^{2 \times 2}$. Moreover, given $\Omega \subset \mathbb{R}^{2}$ open, we define the product space $G_{1}(\Omega):=\Omega \times G_{1,2}$, and endow it with the product distance.

We call varifold any positive Radon measure on $G_{1}(\Omega)$. In this paper we are confined to curves, hence we use the term varifold to mean a 1-varifold.

By varifold convergence we mean the convergence as Radon measures on $G_{1}(\Omega)$.

For any varifold $V$ we define $\mu_{V}$ to be the Radon measure on $\Omega$ obtained projecting $V$ onto $\Omega$.

Let $M$ be a 1-rectifiable subset of $\mathbb{R}^{2}$ and let $\theta: M \rightarrow \mathbb{R}^{+}$be a $\mathcal{H}^{1}\llcorner M$-measurable functions. We define the rectifiable varifold $\mathbf{v}(M, \theta)$, by

$$
\mathbf{v}(M, \theta)(\phi):=\int_{M} \phi\left(x, T_{x} M\right) \theta(x) \mathrm{d} \mathcal{H}^{2} \quad \forall \phi \in C_{c}^{0}\left(G_{1}(\Omega)\right) .
$$

When $\theta$ takes values in $\mathbb{N}$ we say that $\mathbf{v}(M, \theta)$ is a rectifiable integer varifold and we write $\mathbf{v}(M, \theta) \in \mathbf{I V}_{1}(\Omega)$.

Let $V$ be a varifold on $\Omega$. We define the first variation of $V$ as the linear operator

$$
\delta V: C_{c}^{1}\left(\Omega, \mathbb{R}^{2}\right) \rightarrow \mathbb{R}, \quad Y \rightarrow \int \operatorname{tr}(S \nabla Y(x)) \mathrm{d} V(x, S) .
$$

We say that $V$ has bounded first variation if $\delta V$ can be extended to a linear continuous operator on $C_{c}^{0}\left(\Omega, \mathbb{R}^{2}\right)$. In this case by $|\delta V|$ we denote the total variation of $\delta V$. Whenever the varifold $V$ has bounded first variation we call generalized mean curvature vector of $V$ the vector field

$$
\mathbf{H}_{V}=\frac{\mathrm{d} \delta V}{\mathrm{~d} \mu_{V}}
$$

where the right-hand side denotes the Radon-Nikodym derivative of $\delta V$ with respect to $\mu_{V}$. We say that a varifold $V$ is stationary if $\delta V \equiv 0$. We say that $V \in \mathbf{I V}_{1}(\Omega)$ has $L^{2}$-bounded first variation if

$$
\sup _{\substack{Y \in C_{c}^{1}(\Omega),\|Y\|_{L^{2}\left(\mu_{V}\right)} \leq 1}} \delta V(Y)<+\infty .
$$

If $V \in \mathbf{I V}_{1}(\Omega)$ has $L^{2}$-bounded first variation then

$$
\delta V(Y)=\int \mathbf{H}_{V} \cdot Y \mathrm{~d} \mu_{V}, \quad \mathbf{H}_{V} \in L^{2}\left(\mu_{V}, \mathbb{R}^{2}\right),
$$


and we set

$$
\mathcal{F}_{2}(V):=\int\left[1+\left|\mathbf{H}_{V}\right|^{2}\right] \mathrm{d} \mu_{V}=\mu_{V}(\Omega)+\left(\sup _{\substack{Y \in C_{c}^{1}(\Omega),\|Y\|_{L^{2}\left(\mu_{V}\right)} \leq 1}} \delta V(Y)\right)^{2} .
$$

Remark 2.1. If $V \in \mathbf{I V}_{1}(\Omega)$ has $L^{2}$-bounded first variation, by [19], Corollary 17.8, the 1-density of $\mu_{V}$ in $x$

$$
\Theta\left(\mu_{V}, x\right):=\lim _{\rho \rightarrow 0} \frac{\mu_{V}\left(B_{\rho}(x)\right)}{\pi \rho},
$$

is well defined everywhere on $\operatorname{spt}\left(\mu_{V}\right)$. Moreover $\Theta\left(\mu_{V}, x\right) \in \mathbb{N}$ and $\Theta\left(\mu_{V}, x\right)<C$, where $C>0$ is a constant that depends only on $\left\|\mathbf{H}_{\mu_{V}}\right\|_{L^{2}\left(\mu_{V}, \mathbb{R}^{2}\right)}$, furthermore $V=\mathbf{v}(M, \theta)$ where $M=\operatorname{spt}\left(\mu_{V}\right) \cap \Omega$ and $\theta(x)=\Theta\left(\mu_{V}, x\right)$.

For our purposes we also need to define a further class of varifolds, firstly introduced in [14].

Definition 2.2. Let $V \in \mathbf{I V}_{1}(\Omega)$. We say that $V$ is a curvature varifold with generalized second fundamental form in $L^{2}$, if there exists $A_{V}=A_{i j k}^{V} \in L^{2}\left(V, \mathbb{R}^{2^{3}}\right)$ such that for every function $\phi \in C_{c}^{1}\left(G_{1}(\Omega)\right)$ and $i=1,2$,

$$
\int_{G_{1}(\Omega)}\left(\sum_{j=1,2} S_{i j} \partial_{j} \phi+\sum_{j, k=1,2} A_{i j k}^{V} D_{m_{j k}} \phi+\sum_{j=1,2} A_{j i j}^{V} \phi\right) \mathrm{d} V(x, S)=0,
$$

where $D_{m_{j k}} \phi$ denotes the derivative of $\phi(x, \cdot)$ with respect to its $j k$-entry variable.

Moreover we define the generalized second fundamental form $\mathbf{B}_{V}=\left(B_{i j}^{k}\right)_{1 \leq i, j, k \leq 2}$ of $V$ as

$$
B_{i j}^{k}(x, S):=\sum_{l=1}^{2} S_{j l} A_{i k l}^{V}(x, S)
$$

By $\mathscr{C} \mathscr{V}_{1}^{2}(\Omega)$ we denote the class of curvature varifolds in $\Omega$ with generalized second fundamental form in $L^{2}$.

Remark 2.3. Every $V \in \mathscr{C}_{1}^{2}(\Omega)$ has also $L^{2}$-bounded first variation in $\Omega$, and

$$
\mathbf{H}_{V}(x)=\left(A_{212}\left(x, T_{x} \mu_{V}\right), A_{121}\left(x, T_{x} \mu_{V}\right)\right) \in L^{2}\left(\mu_{V}, \mathbb{R}^{2}\right),
$$

for $\mu_{V}$ almost every $x \in \Omega$ (see [14]). Moreover if $V \in \mathscr{C}_{1}^{2}(\Omega)$ we have

$$
\mathcal{F}_{2}(V)=\int\left[1+\left|\mathbf{H}_{V}\right|^{2}\right] \mathrm{d} \mu_{V}=\int\left[1+\left|\mathbf{B}_{V}\right|^{2}\right] \mathrm{d} V=\int\left[1+\left|A_{V}\right|^{2}\right] \mathrm{d} V .
$$

Eventually we need to introduce the following subset of $\mathscr{C} \mathscr{V}_{1}^{2}(\Omega)$

Definition 2.4. We define the set $\mathscr{D}(\Omega)$ as the set of $\mathbf{v}(M, \theta) \in \mathscr{C} \mathscr{V}_{1}^{2}(\Omega)$ for which there exists a sequence $\left\{E_{k}\right\}_{k} \subset \mathscr{C}^{2}(\Omega)$ such that

$$
\lim _{k \rightarrow \infty} \mathbf{v}\left(\partial E_{k}, 1\right)=\mathbf{v}(M, \theta) \text { as varifolds, } \quad \sup _{k \in \mathbb{N}} \mathcal{F}_{2}\left(\mathbf{v}\left(\partial E_{k}, 1\right)\right)<+\infty .
$$

By an adaptation of the results obtained in [2] we prove the following

Proposition 2.5. We have

$$
\mathscr{D}(\Omega)=\left\{\mathbf{v}(M, \theta) \in \mathscr{C} \mathscr{V}_{1}^{2}(\Omega): M \cap \Omega \text { has everywhere an unique tangent line }\right\} .
$$


Proof. For every $n \in \mathbb{N}$, let $\mathcal{Y}_{n}: \Omega \rightarrow \Omega_{n}:=\mathcal{Y}_{n}(\Omega)$ be the map defined by

$$
\mathcal{Y}_{n}(x):=x+\delta_{n}(\operatorname{dist}(x, \partial \Omega)) \nu_{\partial \Omega}\left(\pi_{\partial \Omega}(x)\right),
$$

where: $\delta_{n} \in C^{\infty}(0,+\infty)$ is a decreasing function such that $\delta_{n}(s)=1 / n^{2}$ if $s \in[0,1 / 2 n], \delta_{n}(s)=0$ if $s \geq 1 / n$, and $\left\|\delta_{n}^{\prime}\right\|_{L^{\infty}(0,+\infty)}<10 / n ; \nu_{\partial \Omega}(\cdot)$ denotes the interior unit normal to $\partial \Omega$, and $\pi_{\partial \Omega}(x)$ the projection of $x$ onto $\partial \Omega$. By the regularity assumption on $\Omega$, the map $\mathcal{Y}_{n}$ is a $C^{2}$-diffeomorphism for every $n \in \mathbb{N}$ large enough. Moreover we have $\Omega_{n} \subset \subset \Omega_{n+1} \subset \subset \Omega$, and $\mathcal{Y}_{n}$ converges uniformly to the identity map on $\Omega$ as $n \rightarrow \infty$.

Suppose $\mathbf{v}(M, \theta) \in \mathscr{D}(\Omega)$, and let $\left\{E_{k}\right\}_{k} \subset \mathscr{C}^{2}(\Omega)$ be a sequence verifying (2.8). For a fixed $n \in \mathbb{N}$ the number of connected components of $\partial E_{k}$ such that $\Omega_{n} \cap \partial E_{k} \neq \emptyset$ is bounded by a constant depending only on $n$ and $\sup _{k \in \mathbb{N}} \mathcal{F}_{2}\left(\mathbf{v}\left(\partial E_{k}, 1\right)\right)$. In fact, if the closure $\overline{N_{k}}$ of a connected component of $\partial E_{k}$ intersects $\Omega_{n}$ but does not intersect $\partial \Omega$ then it is a closed curve, and an easy calculation (see [1], Lem. 3.1) shows that the contribution of $N_{k}$ to $\mathcal{F}_{2}\left(\left(\mathbf{v}\left(\partial E_{k}, 1\right)\right)\right.$ is at least $\mathcal{H}^{1}\left(N_{k}\right)+(2 \pi)^{2} / \mathcal{H}^{1}\left(N_{k}\right)$. However, if $N_{k} \cap \Omega_{n} \neq \emptyset$, and $\overline{N_{k}} \cap \partial \Omega \neq \emptyset$, then the contribution of $N_{k}$ to $\mathcal{F}_{2}\left(\left(\mathbf{v}\left(\partial E_{k}, 1\right)\right)\right.$ is larger than $\operatorname{dist}\left(\partial \Omega_{n}, \partial \Omega\right)>0$. Hence, fixed $n \in \mathbb{N}$, we can select a subsequence (not relabeled) such that the connected components of $\partial E_{k}$ intersecting $\Omega_{n}$ are in a fixed, finite number $\Lambda(n)$, and the length of each of this connected components is bounded from below by a constant $C>0$ that depends only on $n$ and $\sup _{k \in \mathbb{N}} \mathcal{F}_{2}\left(\left(\mathbf{v}\left(\partial E_{k}, 1\right)\right)\right.$. Therefore, for every $k \in \mathbb{N}$, and $j=1, \ldots, \Lambda(n)$, we can choose $\alpha_{k}^{j} \in C^{2}\left([0,1], \mathbb{R}^{2}\right)$ such that $\dot{\alpha}_{k}^{j}(t)=$ const. $>C$ for $t \in[0,1]$, and such that $\alpha_{k}^{j}([0,1])=\overline{N_{k}^{j}}$ where $N_{k}^{j}$ is a connected component of $\partial E_{k}$ such that $N_{k}^{j} \cap \Omega_{n} \neq \emptyset$. By (2.8) and

$$
\mathcal{F}_{2}\left(\mathbf{v}\left(N_{k}^{j}, 1\right)\right)=\frac{1}{\left(\mathcal{H}^{1}\left(N_{k}^{j}\right)\right)^{2}} \int_{0}^{1}\left|\ddot{\alpha}_{k}^{j}(t)\right|^{2} \mathrm{~d} t,
$$

we conclude that there exists a subsequence such that, for every $j=1, \ldots \Lambda(n)$, as $k \rightarrow \infty$ the sequence $\alpha_{k}^{j}$ converges weakly in $W^{2,2}\left([0,1], \mathbb{R}^{2}\right)$, and strongly in $C^{1}\left([0,1], \mathbb{R}^{2}\right)$, to a certain constant speed parametrization $\alpha^{j}$ such that

$$
M \cap \Omega_{n}=\cup_{j=1}^{\Lambda(n)} \alpha^{j}([0,1]) \cap \Omega_{n}, \quad \theta(y)=\sum_{i=1}^{\Lambda(n)} \sharp\left\{\left(\alpha^{i}\right)^{-1}(y)\right\} \text { for } \mathcal{H}^{1}-\text { a.e. } y \in \Omega,
$$

where by $\sharp\left\{\left(\alpha^{i}\right)^{-1}(y)\right\}$ we denote the cardinality of the counter-image through $\alpha^{i}$ of $y$.

By construction we have: $\alpha_{k}^{i}([0,1]) \cap \alpha_{k}^{j}([0,1]) \cap \Omega=\emptyset$ for every $i \neq j$ and $k \in \mathbb{N} ; \alpha_{k}^{i}((0,1))$ does not self intersect and if $\alpha_{k}^{i}(0)=\alpha_{k}^{i}(1)$ then $\dot{\alpha}_{k}^{i}(0)=\dot{\alpha}_{k}^{i}(1)$. Hence, by the strong convergence in $C^{1}\left([0,1], \mathbb{R}^{2}\right)$, if for some $s_{0}, s_{1} \in[0,1]$ and $i, j \in\{1, \ldots, \Lambda(n)\}$ we have $\alpha^{i}\left(s_{0}\right)=\alpha^{j}\left(s_{1}\right) \in \Omega_{n}$ then $\dot{\alpha}^{i}\left(s_{0}\right)$ and $\dot{\alpha}^{j}\left(s_{1}\right)$ are parallel. Hence

$$
\mathscr{D}(\Omega) \subseteq\left\{\mathbf{v}(M, \theta) \in \mathscr{C} \mathscr{V}_{1}^{2}(\Omega): M \cap \Omega \text { has everywhere an unique tangent line }\right\} .
$$

In order to prove that also the opposite inclusion holds we proceed as follows. Given $\mathbf{v}(M, \theta) \in \mathscr{C} \mathscr{V}_{1}^{2}(\Omega)$ such that $M \cap \Omega$ has an unique tangent line in every point, we fix $n \in \mathbb{N}$ and consider $\mathbf{v}\left(M \cap \Omega_{n}, \theta\right) \in \mathscr{C}^{2}{ }_{1}^{2}\left(\Omega_{n}\right)$. By [2] we can conclude that $M \cap \Omega_{n}$ can be locally written (up to rigid motions) as a finite union of $W^{2,2}$ graphs, and that $M \cap \partial \Omega_{n}$ consists of a finite number of points. Reasoning as in [1,2], we can find a sequence $\left\{E_{k}^{n}\right\}_{k} \subset \mathscr{C}^{2}\left(\Omega_{n}\right)$ such that

$$
\begin{gathered}
\lim _{k \rightarrow \infty} \mathbf{v}\left(\mathcal{Y}_{n}^{-1}\left(\partial E_{k}^{n}\right), 1\right)=\mathbf{v}\left(\mathcal{Y}_{n}^{-1}\left(M \cap \Omega_{n}\right), \theta\left(\mathcal{Y}_{n}^{-1}\right)\right) \text { as varifolds } \\
\lim _{k \rightarrow \infty} \mathcal{F}_{2}\left(\mathbf{v}\left(\mathcal{Y}_{n}^{-1}\left(\partial E_{k}^{n}\right), 1\right)\right)=\mathcal{F}_{2}\left(\mathbf{v}\left(\mathcal{Y}_{n}^{-1}\left(M \cap \Omega_{n}\right), \theta\left(\mathcal{Y}_{n}^{-1}\right)\right)\right)
\end{gathered}
$$

Since as $n \rightarrow \infty$ we also have

$$
\begin{gathered}
\mathbf{v}\left(\mathcal{Y}_{n}^{-1}\left(M \cap \Omega_{n}\right), \theta\left(\mathcal{Y}_{n}^{-1}\right)\right) \rightarrow \mathbf{v}(M, \theta) \quad \text { as varifolds in } \Omega, \\
\mathcal{F}_{2}\left(\mathbf{v}\left(\mathcal{Y}_{n}^{-1}\left(M \cap \Omega_{n}\right), \theta\left(\mathcal{Y}_{n}^{-1}\right)\right)\right) \rightarrow \mathcal{F}_{2}(\mathbf{v}(M, \theta)),
\end{gathered}
$$


we can extract a diagonal sequence $\left\{\mathcal{Y}_{n}^{-1}\left(E_{k(n)}^{n}\right)\right\}_{n} \subset \mathscr{C}^{2}(\Omega)$ verifying (2.8). Hence

$$
\mathscr{D}(\Omega) \supseteq\left\{\mathbf{v}(M, \theta) \in \mathscr{C} \mathscr{V}_{1}^{2}(\Omega): M \cap \Omega \text { has everywhere an unique tangent line }\right\}
$$

and this concludes the proof.

Finally, being $\overline{\mathcal{F}}$ as in (1.12), as a straightforward consequence of [2], Theorem 4.3, and Proposition 2.5 we have the following

Theorem 2.6. Let $u \in L^{1}(\Omega)$. Then $\overline{\mathcal{F}}(u)<+\infty$ if and only if $u=\mathbb{1}_{E} \in B V(\Omega,\{-1,1\})$, and, if $E \neq \emptyset$, the set

$$
\begin{aligned}
\mathscr{A}(E):=\{\mathbf{v}(M, \theta) \in \mathscr{D}(\Omega): & M \supset \partial^{*} E, \\
& \theta(x) \text { is odd for } x \in \partial^{*} E, \\
& \left.\theta(x) \text { is even for } x \in \operatorname{spt}\left(\mu_{V}\right) \backslash \partial^{*} E\right\},
\end{aligned}
$$

is not empty. Moreover, if $\mathscr{A}(E) \neq \emptyset$, the following representation formula holds

$$
\overline{\mathcal{F}}\left(\mathbb{1}_{E}\right)=\min _{\mathbf{v}(M, \theta) \in \mathscr{A}(E)} \mathcal{F}_{2}(\mathbf{v}(M, \theta)) .
$$

In particular if $E \in \mathscr{W}^{2,2}(\Omega)$ we have $\overline{\mathcal{F}}\left(\mathbb{1}_{E}\right)=\mathcal{F}_{2}(\mathbf{v}(\partial E, 1))$.

\section{Preliminary known Results on diffuse interfaces approximations of $\mathcal{F}$}

We begin this section specifying some further notation needed in the sequel.

We set $W(r):=\frac{1}{4}\left(1-r^{2}\right)^{2}$ for $r \in \mathbb{R}$, and $c_{0}:=\int_{-1}^{1} \sqrt{2 W(s)} \mathrm{d} s$.

To every family $\left\{u_{\varepsilon}\right\}_{\varepsilon} \subset C^{2}(\Omega)$ we associate

- the families of Radon measures

$$
\mu_{\varepsilon}:=\left(\frac{\varepsilon}{2}\left|\nabla u_{\varepsilon}\right|^{2}+\frac{W\left(u_{\varepsilon}\right)}{\varepsilon}\right) \mathcal{L}_{L}^{2}, \quad \widetilde{\mu}_{\varepsilon}:=\varepsilon\left|\nabla u_{\varepsilon}\right|^{2} \mathcal{L}_{L}^{2} \Omega ;
$$

- the family of diffuse varifolds

$$
V_{\varepsilon}(\phi):=c_{0}^{-1} \int \phi\left(x, P^{u_{\varepsilon}}(x)\right) d \widetilde{\mu}_{\varepsilon}(x), \quad \forall \phi \in C_{c}^{0}\left(G_{1}(\Omega)\right),
$$

where $P^{u_{\varepsilon}}(x)$ denotes the projection on the tangent space to the level line of $u_{\varepsilon}$ passing through $x$ (see $(2.2)$ ).

The next result has been proved in $[17,18]$

Theorem 3.1. Let $\left\{u_{\varepsilon}\right\} \subset C^{2}(\Omega)$ be a family such that

$$
\sup _{\varepsilon>0} \widetilde{\mathcal{E}}_{\varepsilon}\left(u_{\varepsilon}\right)=\sup _{\varepsilon>0}\left(\mathcal{P}_{\varepsilon}\left(u_{\varepsilon}\right)+\mathcal{W}_{\varepsilon}\left(u_{\varepsilon}\right)\right)<+\infty .
$$

(A) There exists a subsequence (still denoted by $\left\{u_{\varepsilon}\right\}$ ) converging in $L^{1}(\Omega)$ to a function $\mathbb{1}_{E} \in B V(\Omega,\{-1,1\})$. Moreover the sequence $\left\{V_{\varepsilon}\right\}_{\varepsilon}$ converges in the varifolds sense to $\mathbf{v}(M, \theta) \in \mathbf{I V}_{1}(\Omega)$ with $L^{2}$-bounded first variation, such that $\theta$ assumes odd (respectively even) values on $\partial^{*} E$ (respectively $M \backslash \partial^{*} E$ ) and

$$
\begin{gathered}
c_{0} \theta \mathcal{H}_{L_{M}^{1}}^{1}=\lim _{\varepsilon \rightarrow 0^{+}} \mu_{\varepsilon}=\lim _{\varepsilon \rightarrow 0^{+}} \widetilde{\mu}_{\varepsilon} \text { as Radon measures, } \\
\liminf _{\varepsilon \rightarrow 0^{+}} \frac{1}{\varepsilon} \int_{\Omega}\left(\varepsilon \Delta u_{\varepsilon}-\frac{W^{\prime}\left(u_{\varepsilon}\right)}{\varepsilon}\right)^{2} \mathrm{~d} x \geq c_{0} \int\left|\mathbf{H}_{V}\right|^{2} \mathrm{~d} \mu_{V} .
\end{gathered}
$$


(B) For every $\mathbb{1}_{E} \in B V(\Omega,\{-1,1\})$ such that $E \in \mathscr{W}^{2,2}(\Omega)$, we have

$$
\Gamma\left(L^{1}(\Omega)\right)-\lim _{\varepsilon \rightarrow 0} \widetilde{\mathcal{E}}_{\varepsilon}\left(\mathbb{1}_{E}\right)=c_{0} \mathcal{F}\left(\mathbb{1}_{E}\right)
$$

Next we recall some of the main results obtained in [3].

Theorem 3.2. Let $\left\{u_{\varepsilon}\right\} \subset C^{2}(\Omega)$ be such that

$$
\sup _{\varepsilon>0} \mathcal{E}_{\varepsilon}\left(u_{\varepsilon}\right):=\sup _{\varepsilon>0}\left(\mathcal{P}_{\varepsilon}\left(u_{\varepsilon}\right)+\mathcal{B}_{\varepsilon}\left(u_{\varepsilon}\right)\right)<+\infty .
$$

(A1) The conclusions of Theorem 3.1 hold. Moreover $\mathbf{v}(M, \theta) \in \mathscr{C}^{2}{ }_{1}^{2}(\Omega)$ and

$$
\liminf _{\varepsilon \rightarrow 0^{+}} \frac{1}{\varepsilon} \int_{\Omega}\left|\varepsilon \nabla^{2} u_{\varepsilon}-\frac{W^{\prime}\left(u_{\varepsilon}\right)}{\varepsilon} \nu_{u_{\varepsilon}} \otimes \nu_{u_{\varepsilon}}\right|^{2} \mathrm{~d} x \geq c_{0} \int\left|\mathbf{B}_{V}\right|^{2} \mathrm{~d} V .
$$

(B1) For every $\mathbb{1}_{E} \in B V(\Omega,\{-1,1\})$ such that $E \in \mathscr{W}^{2,2}(\Omega)$, we have

$$
\Gamma\left(L^{1}(\Omega)\right)-\lim _{\varepsilon \rightarrow 0} \mathcal{E}_{\varepsilon}\left(\mathbb{1}_{E}\right)=c_{0} \int_{\Omega \cap \partial E}\left[1+\left|\mathbf{B}_{\partial E}\right|^{2}\right] \mathrm{d} \mathcal{H}^{1}=c_{0} \mathcal{F}\left(\mathbb{1}_{E}\right) .
$$

\section{Main Results}

The first of our main results shows that a varifold $\mathbf{v}(M, \theta) \in \mathscr{C} \mathscr{V}_{1}^{2}(\Omega)$ arising as the limit of diffuse interface varifolds veryfing (3.6) (see Thm. 3.2-(A1)) is more regular than a generic element of $\mathscr{C}_{1}^{2}(\Omega)$.

Theorem 4.1. Let $\left\{u_{\varepsilon}\right\}_{\varepsilon} \subset C^{2}(\Omega)$ satisfy (3.6). Let $V_{\varepsilon}$ be as in (3.2) and suppose $\lim _{\varepsilon \rightarrow 0} V_{\varepsilon}=\mathbf{v}(M, \theta) \in$ $\mathscr{C V}_{1}^{2}(\Omega)$. Then $M$ has an unique tangent line in every $p \in M \cap \Omega$.

In order to prove Theorem 4.1 we need two easy Lemmata.

Lemma 4.2. Let $\left\{M_{k}\right\}_{k} \subset B_{2 R}$ be a sequence of $C^{2}$-embedded curves without boundary in $B_{2 R}$. Suppose that

$$
\begin{gathered}
0<\liminf _{k \rightarrow \infty} \mathcal{H}^{1}\left(M_{k} \cap B_{R}\right), \quad \limsup _{k \rightarrow \infty} \mathcal{H}^{1}\left(M_{k}\right)<+\infty, \\
\lim _{k \rightarrow \infty}\left|\delta \mathbf{v}\left(M_{k}, 1\right)\right|\left(B_{2 R}\right)=\lim _{k \rightarrow \infty} \int_{M_{k}}\left|\mathbf{H}_{M_{k}}\right| \mathrm{d} \mathcal{H}^{1}=0 .
\end{gathered}
$$

There exist a finite collection of 1-dimensional affine subspaces $T_{1}, \ldots, T_{N}$ of $\mathbb{R}^{2}$ such that

$$
T_{i} \cap T_{j} \cap B_{R}=\emptyset, \quad \text { for } i \neq j, i, j \in\{1, \ldots, N\},
$$

and a subsequence (not relabelled) $\left\{\mathbf{v}\left(M_{k}, 1\right)\right\}_{k} \subset \mathbf{I V}_{1}\left(B_{2 R}\right)$ such that

$$
\lim _{k \rightarrow \infty} \mathbf{v}\left(M_{k}, 1\right)=\sum_{j=1}^{N} \mathbf{v}\left(T_{j}, \Theta_{j}\right)
$$

where $\Theta_{j} \in \mathbb{N}$ are constants.

Proof. By (4.1) we can apply Allard's compactness Theorem (see [19], Thm. 42.7), and extract a subsequence such that, as $k \rightarrow \infty, \mathbf{v}\left(M_{k}, 1\right) \rightarrow \mathbf{v}(M, \theta) \in \mathbf{I V}_{1}\left(B_{2 R}\right)$, with $\mathbf{v}(M, \theta)$ stationary in $B_{2 R}$, and $\mu_{V}\left(B_{R}\right)>0$. 
By (4.1), and arguing as in the proof of Lemma 2.5, we select a further subsequence (not relabeled) such that:

(i) there are no closed curves among the connected components of $M_{k}$;

(ii) the connected components of $M_{k}$ intersecting $B_{3 R / 2}$ are in a fixed number.

Hence we can find a constant $C>0$, and $N$ sequences of maps $\left\{\alpha_{k}^{j}\right\}_{k \in \mathbb{N}} \subset C^{2}\left([0,1], \overline{B_{3 R / 2}}\right)$ such that, for $j=1, \ldots, N$ and $k \in \mathbb{N}$, we have

$$
C<\left|\dot{\alpha}_{k}^{j}\right|=\text { const. on }[0,1], \quad M_{k} \cap B_{R}=\bigcup_{j=1}^{N}\left(\alpha_{k}^{j}\right)([0,1]) \cap B_{R} .
$$

Since

$$
\lim _{k \rightarrow \infty} \sum_{j=1}^{N} \frac{1}{\mathcal{H}^{1}\left(\alpha_{k}^{j}(0,1)\right)} \int_{0}^{1}\left|\ddot{\alpha}_{k}^{j}\right| \mathrm{d} t \leq \lim _{k \rightarrow \infty}\left|\delta \mathbf{v}\left(M_{k}, 1\right)\right|\left(B_{2 R}\right)=0,
$$

(up to the extraction of a further subsequence) $\alpha_{k}^{j} \rightarrow \alpha^{j}$ strongly in $W^{2,1}\left([0,1], \mathbb{R}^{2}\right)$, for every $j=1, \ldots, N$; $\alpha_{k}^{j} \rightarrow \alpha^{j}$ in $C^{1}\left([0,1] ; \mathbb{R}^{2}\right)$ and $\ddot{\alpha}^{j}=0$ on $[0,1]$. Since for every $\phi \in C_{c}^{0}\left(G_{1}\left(B_{R}\right)\right)$

$$
\begin{aligned}
\lim _{k \rightarrow \infty} \mathbf{v}\left(M_{k}, 1\right)(\phi) & =\lim _{k \rightarrow \infty} \sum_{j=1}^{N} \int_{0}^{1} \phi\left(\alpha_{k}^{j}(s), I d-\frac{\dot{\alpha}_{k}^{j}(s) \otimes \dot{\alpha}_{k}^{j}(s)}{\left|\dot{\alpha}_{k}^{j}(s)\right|^{2}}\right)\left|\dot{\alpha}_{k}^{j}(s)\right| \mathrm{d} s \\
& =\sum_{j=1}^{N} \int_{0}^{1} \phi\left(\alpha^{j}(s), I d-\frac{\dot{\alpha}^{j}(s) \otimes \dot{\alpha}^{j}(s)}{\left|\dot{\alpha}^{j}(s)\right|^{2}}\right)\left|\dot{\alpha}^{j}(s)\right| \mathrm{d} s,
\end{aligned}
$$

we conclude that (4.3) holds for $T_{j} \cap B_{R}:=\alpha^{j}([0,1]) \cap B_{R}$. Finally (4.2) follows, as in Proposition 2.5, by the strong convergence $\alpha_{k}^{j} \rightarrow \alpha_{j}$ in $C^{1}\left([0,1], \mathbb{R}^{2}\right)$ and $\alpha_{k}^{j}([0,1]) \cap \alpha_{k}^{l}([0,1])=\emptyset$ for every $j \neq l \in\{1, \ldots, N\}$ and $k \in \mathbb{N}$.

Lemma 4.3. Let $\widetilde{u}_{\varepsilon} \in C^{2}\left(B_{2 R}\right)$ be such that

$$
\begin{gathered}
0<\lim _{\varepsilon \rightarrow 0} \int_{B_{2 R}} \frac{\varepsilon}{2}\left|\nabla \widetilde{u}_{\varepsilon}\right|^{2}+\frac{W\left(\widetilde{u}_{\varepsilon}\right)}{\varepsilon} \mathrm{d} x<+\infty, \\
\lim _{\varepsilon \rightarrow 0} \frac{1}{\varepsilon} \int_{B_{2 R}}\left|\varepsilon \nabla^{2} \widetilde{u}_{\varepsilon}-\frac{W^{\prime}\left(\widetilde{u}_{\varepsilon}\right)}{\varepsilon} \nu_{\widetilde{u}_{\varepsilon}} \otimes \nu_{\widetilde{u}_{\varepsilon}}\right|^{2} \mathrm{~d} x=0 .
\end{gathered}
$$

Being $\widetilde{V}_{\varepsilon}$ the diffuse interface varifold associated to $\widetilde{u}_{\varepsilon}$ (see (3.2)), up to a subsequence we have $\lim _{\varepsilon \rightarrow 0} \widetilde{V}_{\varepsilon}=\widetilde{V}$, where $\widetilde{V} \in \mathbf{I V}_{1}\left(B_{2 R}\right)$ is stationary and verifies (4.3) and (4.2).

Proof. By (4.4), (4.5) we can apply Theorem 3.2, and extract a subsequence (not relabeled) such that $\widetilde{V}_{\varepsilon} \rightarrow \widetilde{V} \in$ $\mathscr{C} \mathscr{V}_{1}^{2}\left(B_{2 R}\right)$, where $\widetilde{V}$ is stationary. Moreover by Sard's Lemma and [3], Lemma 7.1, we can find a subsequence $\left\{\widetilde{V}_{\varepsilon_{k}}\right\}_{k}$ and a subset $J \subset[-1,1]$, with $\mathcal{L}^{1}(J)=0$, such that for every $s \in[-1,1] \backslash J$,

$\left\{\widetilde{u}_{\varepsilon_{k}}=s\right\}$ is a smooth embedded surface without boundary in $B_{2 R}$

$$
\begin{gathered}
\left\{\widetilde{u}_{\varepsilon_{k}}=s\right\} \cap\left\{\nabla \widetilde{u}_{\varepsilon_{k}}=0\right\}=\emptyset, \\
\lim _{k \rightarrow \infty} \mathbf{v}\left(\left\{\widetilde{u}_{\varepsilon_{k}}=s\right\}, 1\right)=\widetilde{V} \text { as varifolds on } B_{2 R} .
\end{gathered}
$$


Next we fix $\delta \in(0,1)$ and set $I_{\delta}:=[-1+\delta, 1-\delta]$. For every $x \in B_{2 R}$ such that $\widetilde{u}_{\varepsilon_{k}}(x)=s \in[-1,1] \backslash J$ let $\mathbf{B}_{\widetilde{u}_{\varepsilon_{k}}}(x)$ be as in (2.3). As in [3], Lemma 5.3, we have

$$
\begin{aligned}
& \int_{I_{\delta} \backslash J}\left|\delta \mathbf{v}\left(\left\{\widetilde{u}_{\varepsilon_{k}}=s\right\}, 1\right)\right|\left(B_{2 R}\right) \mathrm{d} s=\int_{I_{\delta} \backslash J} \int_{\left\{\widetilde{u}_{\varepsilon_{k}}=s\right\} \cap B_{2 R}}\left|\operatorname{div}\left(\nu_{\widetilde{u}_{\varepsilon_{k}}}\right)\right| \mathrm{d} \mathcal{H}^{1} \mathrm{~d} s \\
\leq & \frac{2}{\left(2 \delta-\delta^{2}\right)} \int_{B_{2 R}}\left|\mathbf{B}_{\widetilde{u}_{\varepsilon_{k}}}\right| \sqrt{2 W\left(\widetilde{u}_{\varepsilon_{k}}\right)}\left|\nabla \widetilde{u}_{\varepsilon_{k}}\right| \mathrm{d} x \\
\leq & \frac{2}{\left(2 \delta-\delta^{2}\right)}\left(\frac{1}{\varepsilon_{k}} \int_{B_{2 R}}\left|\varepsilon \nabla^{2} \widetilde{u}_{\varepsilon}-\frac{W^{\prime}\left(\widetilde{u}_{\varepsilon}\right)}{\varepsilon} \nu \widetilde{u}_{\varepsilon} \otimes \nu \widetilde{u}_{\varepsilon}\right|^{2} \mathrm{~d} x\right)^{1 / 2}\left(\int_{B_{2 R}} \frac{W\left(\widetilde{u}_{\varepsilon_{k}}\right)}{\varepsilon_{k}} \mathrm{~d} x\right)^{1 / 2} .
\end{aligned}
$$

By (4.5), the choice of $\varepsilon_{k}$ and that of the set $J$, there exists $s_{\varepsilon_{k}} \in I_{\delta} \backslash J$ such that

$$
\limsup _{k \rightarrow \infty} \mathcal{H}^{1}\left(\left\{\widetilde{u}_{\varepsilon_{k}}=s_{\varepsilon_{k}}\right\} \cap B_{2 R}\right)<+\infty, \quad \limsup _{k \rightarrow \infty}\left|\delta \mathbf{v}\left(\left\{\widetilde{u}_{\varepsilon_{k}}=s_{\varepsilon_{k}}\right\}, 1\right)\right|\left(B_{2 R}\right)=0 .
$$

Applying Lemma 4.2 to the sequence $\left\{\mathbf{v}\left(\left\{\widetilde{u}_{\varepsilon_{k}}=s_{\varepsilon_{k}}\right\}, 1\right)\right\}_{k} \subset \mathbf{I V}_{1}\left(B_{2 R}\right)$, and making use of (4.6), we conclude the proof.

Proof of Theorem 4.1. For $x \in \mathbb{R}^{2}$ and $\lambda>0$ we define

$$
\eta_{x, \lambda}: \mathbb{R}^{2} \rightarrow \mathbb{R}^{2}, \quad y \mapsto \frac{y-x}{\lambda},
$$

and consider, for $x \in \operatorname{spt}\left(\mu_{V}\right)$, the varifolds

$$
\left(\eta_{x, \rho}\right)_{\sharp} V(\phi):=\frac{1}{\rho} \int \phi(\rho y+x, Q) \mathrm{d} V(y, Q), \quad \forall \phi \in C_{c}^{0}\left(G_{1}\left(\mathbb{R}^{2}\right)\right) .
$$

By [13], Theorem 3.4 we can conclude that for every $x \in \operatorname{spt}\left(\mu_{V}\right)$ there exists $V_{x} \in \mathbf{I V}_{1}\left(\mathbb{R}^{2}\right)$ such that

$$
\lim _{\rho \rightarrow 0^{+}}\left(\eta_{x, \rho}\right)_{\sharp} V(\phi)=V_{x}(\phi), \quad \forall \phi \in C_{c}^{0}\left(G_{1}\left(\mathbb{R}^{2}\right)\right),
$$

and

$$
V_{x}=\sum_{i=1}^{N_{x}} \mathbf{v}\left(T_{i}(x), \Theta_{i}(x)\right),
$$

where $N_{x} \in \mathbb{N}$, and where $\tilde{T}_{1}(x), \ldots, \tilde{T}_{N_{x}}(x) \in G_{1,2}$ and $\Theta_{1}(x), \ldots, \Theta_{N_{x}}(x) \in \mathbb{N}$ verify

$$
\bigcap_{i=1}^{N_{x}} T_{i}(x)=\{0\}, \quad \sum_{i=1}^{N_{x}} \Theta_{i}(x)=\theta(x) .
$$

In order to prove the existence of an unique tangent line in every point of $\operatorname{spt}\left(\mu_{V}\right)$ we show that $N_{x}=1$ for every $x \in \operatorname{spt}\left(\mu_{V}\right)$.

Without loss of generality we suppose that $x=0$. In view of (4.7) to conclude that $N_{0}=1$ it is enough to fix a sequence $\left\{\rho_{k}\right\}_{k} \subset \mathbb{R}^{+}$such that $\lim _{k \rightarrow \infty} \rho_{k}=0$, and prove that

$$
V_{0}=\lim _{k \rightarrow \infty}\left(\eta_{0, \rho_{k}}\right)_{\sharp} V=\mathbf{v}(T, \theta(0)),
$$

where $T \in G_{1,2}$ is a linear 1-dimensional subspace of $\mathbb{R}^{2}$. 
By (4.7), (3.6) and by $\lim _{k \rightarrow \infty} V_{\varepsilon_{k}}=V$ as varifolds in $\Omega$, fixed an open bounded subset $U \subset \mathbb{R}^{2}$ containing the origin, we can find a sequence $\left\{\varepsilon_{k}\right\}_{k}$ such that

$$
\lim _{k \rightarrow \infty} \varepsilon_{k}=\lim _{k \rightarrow \infty} \frac{\varepsilon_{k}}{\rho_{k}}=0,
$$

and such that, setting $\widetilde{u}_{k}(y):=u_{\varepsilon_{k}}\left(\rho_{k} y\right), \widetilde{\varepsilon}_{k}:=\varepsilon_{k} / \rho_{k}$, denoted by $\widetilde{V}_{\widetilde{\varepsilon}_{k}}$ the diffuse varifolds associated to $\widetilde{u}_{k}$ as in (3.2) we have

$$
\widetilde{V}_{\widetilde{\varepsilon}_{k}} \rightarrow V_{0} \quad \text { as varifolds in } U, \quad 0<\lim _{k \rightarrow \infty} \int_{U} \widetilde{\varepsilon}_{k}\left|\nabla \widetilde{u}_{k}\right|^{2}+\frac{W\left(\widetilde{u}_{k}\right)}{\widetilde{\varepsilon}_{k}} \mathrm{~d} x<+\infty
$$

and, by (2.7),

$$
\begin{gathered}
\frac{1}{\widetilde{\varepsilon}_{k}} \int_{U}\left|\widetilde{\varepsilon}_{k} \nabla^{2} \widetilde{u}_{k}-\frac{W^{\prime}\left(\widetilde{u}_{k}\right)}{\widetilde{\varepsilon}_{k}} \nu_{\widetilde{u}_{k}} \otimes \nu_{\widetilde{u}_{k}}\right|^{2} \mathrm{~d} y \\
=\frac{\rho_{k}}{\varepsilon_{k}} \int_{\rho_{k} U}\left|\varepsilon_{k} \nabla^{2} u_{\varepsilon_{k}}(x)-\frac{W^{\prime}\left(u_{\varepsilon_{k}}(x)\right)}{\varepsilon_{k}} \nu_{u_{\varepsilon_{k}}}(x) \otimes \nu_{u_{\varepsilon_{k}}}(x)\right|^{2} \mathrm{~d} x \leq C \rho_{k} .
\end{gathered}
$$

We can thus apply Lemma 4.3 and obtain that

$$
\widetilde{V}_{\widetilde{\varepsilon}_{k}} \rightarrow V_{0}=\sum_{j=1}^{N_{0}} \mathbf{v}\left(T_{j} \cap U, \Theta_{j}\right) \quad \text { as varifolds in } U,
$$

where, if $N_{0}>1$, we have $T_{i} \cap T_{j} \cap B_{R}=\emptyset$ for every $B_{2 R} \subset \subset U$ and $i \neq j$. Hence, by (4.8), we have $N_{0}=1$.

As a consequence of Theorems 4.1 and 2.6 we obtain

Corollary 4.4. We have $\Gamma\left(L^{1}(\Omega)\right)-\lim _{\varepsilon \rightarrow 0} \mathcal{E}_{\varepsilon}=c_{0} \overline{\mathcal{F}}$, where $\overline{\mathcal{F}}$ is as in $(1.12)$.

Proof. We begin proving the so-called $\Gamma-\lim$ inf-inequality, that is: For every $u_{\varepsilon} \rightarrow u$ in $L^{1}(\Omega)$ as $\varepsilon \rightarrow 0$ we have

$$
\liminf _{\varepsilon \rightarrow 0} \mathcal{E}_{\varepsilon}\left(u_{\varepsilon}\right) \geq c_{0} \overline{\mathcal{F}}(u)
$$

We suppose that $\left\{u_{\varepsilon}\right\}_{\varepsilon} \subset C^{2}(\Omega)$ satisfies (3.6) (otherwise we have nothing to prove). By Theorem 3.2 we can find a subsequence $\left\{\varepsilon_{k}\right\}_{k \in \mathbb{N}}$ such that $\lim _{k \rightarrow \infty} \varepsilon_{k}=0$ and

$$
\begin{gathered}
\lim _{k \rightarrow \infty} \mathcal{E}_{\varepsilon_{k}}\left(u_{\varepsilon_{k}}\right)=\liminf _{\varepsilon \rightarrow 0} \mathcal{E}_{\varepsilon}\left(u_{\varepsilon}\right), \\
L^{1}(\Omega)-\lim _{k \rightarrow \infty} u_{\varepsilon_{k}} \rightarrow \mathbb{1}_{E} \in B V(\Omega,\{-1,1\}), \quad \lim _{k \rightarrow \infty} V_{\varepsilon_{k}}=\mathbf{v}(M, \theta) \in \mathscr{C} \mathscr{V}_{1}^{2}(\Omega) \text { as varifolds. }
\end{gathered}
$$

Moreover, by Proposition 2.5 and Theorem 4.1, we have $\mathbf{v}(M, \theta) \in \mathscr{D}(\Omega)$. Hence, by Theorem 2.6, Theorem 3.2-(A1) and (2.7),

$$
\liminf _{\varepsilon \rightarrow 0} \mathcal{E}_{\varepsilon}\left(u_{\varepsilon}\right)=\lim _{k \rightarrow \infty} \mathcal{E}_{\varepsilon_{k}}\left(u_{\varepsilon_{k}}\right) \geq c_{0} \int_{M}\left(1+\left|\mathbf{B}_{V}\right|^{2}\right) \theta \mathrm{d} \mathcal{H}^{1} \geq c_{0} \overline{\mathcal{F}}(E) .
$$

That is the $\Gamma-\lim$ inf inequality holds.

To prove the $\Gamma-\lim$ sup inequality we have to show that for every $u \in L^{1}(\Omega)$ we can find a recovery family, that is a family $\left\{u_{\varepsilon}\right\}_{\varepsilon} \subset C^{2}(\Omega)$ verifying

$$
\lim _{\varepsilon \rightarrow 0}\left\|u_{\varepsilon}-u\right\|_{L^{1}(\Omega)}=0, \quad \limsup _{\varepsilon \rightarrow 0} \mathcal{E}_{\varepsilon}\left(u_{\varepsilon}\right) \leq \overline{\mathcal{F}}(u) .
$$


However, this now follows by Proposition 2.5 and a standard density argument. In fact, by the previous step we can conclude that for every $u \in L^{1}(\Omega)$ such that $\Gamma\left(L^{1}(\Omega)\right)-\lim _{\varepsilon \rightarrow 0} \mathcal{E}_{\varepsilon}(u)<+\infty$ we also have $u=\mathbb{1}_{E}$ and $\overline{\mathcal{F}}\left(\mathbb{1}_{E}\right)<+\infty$. Therefore we can find a sequence $\left\{E_{k}\right\}_{k} \subset \mathscr{C}^{2}(\Omega)$ such that

$$
L^{1}(\Omega)-\lim _{k \rightarrow \infty} \mathbb{1}_{E_{k}}=\mathbb{1}_{E}, \quad \lim _{k \rightarrow \infty} \mathcal{F}\left(\mathbb{1}_{E_{k}}\right)=\overline{\mathcal{F}}\left(\mathbb{1}_{E}\right) .
$$

Since for any fixed $k \in \mathbb{N}$ the existence of a recovery family $\left\{u_{\varepsilon}^{k}\right\}_{\varepsilon}$ follows from [3,4], we can extract a diagonal sequence verifying (4.10).

Eventually we also obtain a results concerning the $\Gamma$-limit of the family of functionals $\left\{\widetilde{\mathcal{E}}_{\varepsilon}\right\}_{\varepsilon}$, and its relation with $\overline{\mathcal{F}}$. More precisely, combining Corollary 4.4 with the results proved in [8] (see also [6]), we have

Corollary 4.5. There exists a family $\left\{u_{\varepsilon}\right\}_{\varepsilon} \subset C^{2}(\Omega)$ and $\mathbb{1}_{E} \in B V(\Omega,\{-1,1\})$, where $E \neq \emptyset$ is such that $\partial E$ does not have an unique tangent line in every point, verifying

$$
\begin{gathered}
L^{1}(\Omega)-\lim _{\varepsilon \rightarrow 0} u_{\varepsilon}=\mathbb{1}_{E}, \quad \lim _{\varepsilon \rightarrow 0} V_{\varepsilon}=\mathbf{v}(\partial E, 1) \in \mathscr{C}_{1}^{2}(\Omega), \\
\sup _{\varepsilon>0} \mathcal{P}_{\varepsilon}\left(u_{\varepsilon}\right)<+\infty, \quad \mathcal{W}_{\varepsilon}\left(u_{\varepsilon}\right) \equiv 0 .
\end{gathered}
$$

In particular

$$
\lim _{\varepsilon \rightarrow 0} \widetilde{\mathcal{E}}_{\varepsilon}\left(u_{\varepsilon}\right)=\mathcal{F}_{2}(\mathbf{v}(\partial E, 1))<\overline{\mathcal{F}}\left(\mathbb{1}_{E}\right)=\Gamma\left(L^{1}(\Omega)\right)-\lim _{\varepsilon \rightarrow 0} \mathcal{E}_{\varepsilon}\left(\mathbb{1}_{E}\right)=+\infty .
$$

Proof. By [6], Theorem 1.3 (see also [8]) we can find $U \in C^{3}\left(\mathbb{R}^{2}\right)$ such that

$$
\Delta U=W^{\prime}(U) \quad \text { on } \mathbb{R}^{2},
$$

and $U$ is such that

- $\|U\|_{L^{\infty}\left(\mathbb{R}^{2}\right)} \leq 1,\{U=0\}=\mathfrak{C}$ where

$$
\mathfrak{C}:=(\{0\} \times \mathbb{R}) \cup(\mathbb{R} \times\{0\})
$$

and $U>0$ (respectively $U<0$ ) in the I and III (respectively II and IV) quadrant of $\mathbb{R}^{2}$;

- there exists $C>0$ such that for every $R>0$

$$
\int_{B_{R}} \frac{1}{2}|\nabla U|^{2}+W(U) \mathrm{d} y \leq C R
$$

Defining $\left\{u_{\varepsilon}\right\}_{\varepsilon} \subset C^{2}(\Omega)$ by $u_{\varepsilon}(x):=U(x / \varepsilon)$, by (4.12), (4.13) we have

$$
\begin{gathered}
\varepsilon \Delta u_{\varepsilon}-\frac{W^{\prime}\left(u_{\varepsilon}\right)}{\varepsilon}=0, \\
\int_{\Omega} \frac{\varepsilon}{2}\left|\nabla u_{\varepsilon}\right|^{2}+\frac{W\left(u_{\varepsilon}\right)}{\varepsilon} \mathrm{d} x=\varepsilon \int_{B_{\varepsilon}-1} \frac{1}{2}|\nabla U|^{2}+W(U) \mathrm{d} y \leq C .
\end{gathered}
$$

Hence applying Theorem 3.1 we have that, up to subsequences, $u_{\varepsilon} \rightarrow \mathbb{1}_{E} \in B V(\Omega,\{-1,1\})$ and $V_{\varepsilon} \rightarrow \mathbf{v}(M, \theta) \in$ $\mathbf{I V}_{1}(\Omega)$, where $\mathbf{v}(M, \theta)$ is stationary in $\Omega$. Moreover, in view of [8], Lemma 5 , and [18], we obtain that $E$ coincides with the intersection of $\Omega$ with the $I, I I I$ quadrants of $\mathbb{R}^{2}$, and also that $M=\mathfrak{C}=\partial E$. This concludes the proof of the first part of Corollary 4.5. It remains to prove that (4.11) holds. To this aim it is enough to remark that, being $\left\{u_{\varepsilon}\right\}_{\varepsilon}$ and $\mathbb{1}_{E}$ as above, by Proposition 2.5 and Theorem 4.1 we have

$$
\overline{\mathcal{F}}\left(\mathbb{1}_{E}\right)=\Gamma\left(L^{1}(\Omega)\right)-\lim _{\varepsilon \rightarrow 0} \mathcal{E}_{\varepsilon}\left(\mathbb{1}_{E}\right)=+\infty .
$$


Remark 4.6. We remark that combining Corollary 4.5 with [2], Example 1, we obtain the existence of an open subset $E \subset \subset B_{10}$ and of a family $\left\{u_{\varepsilon}\right\}_{\varepsilon} \subset C^{2}\left(B_{10}\right)$ such that

$$
\begin{aligned}
L^{1}\left(B_{10}\right)-\lim _{\varepsilon \rightarrow 0} u_{\varepsilon}= & \mathbb{1}_{E}, \quad \lim _{\varepsilon \rightarrow 0} V_{\varepsilon}=\mathbf{v}(M, \theta) \in \mathscr{C} \mathscr{V}_{1}^{2}\left(B_{10}\right) \backslash \mathscr{A}(E), \\
& \lim _{\varepsilon \rightarrow 0} \widetilde{\mathcal{E}}_{\varepsilon}\left(u_{\varepsilon}\right)<\overline{\mathcal{F}}\left(\mathbb{1}_{E}\right)<+\infty .
\end{aligned}
$$

Acknowledgements. I wish to thank Giovanni Bellettini, Andreas Rätz and Matthias Röger for several interesting discussions on the subject of this paper.

\section{REFERENCES}

[1] G. Bellettini, G. Dal Maso and M. Paolini, Semicontinuity and relaxation properties of a curvature depending functional in 2d. Ann. Scuola Norm. Sup. Pisa Cl. Sci. 20 (1993) 247-297.

[2] G. Bellettini and L. Mugnai, A varifolds representation of the relaxed elastica functional. J. Convex Anal. 14 (2007) 543-564.

[3] G. Bellettini and L. Mugnai, Approximation of Helfrich's functional via diffuse interfaces. SIAM J. Math. Anal. 42 (2010) 2402-2433.

[4] G. Bellettini and M. Paolini, Approssimazione variazionale di funzionali con curvatura. Seminario Analisi Matematica Univ. Bologna (1993).

[5] A. Braides and R. March, Approximation by $\Gamma$-convergence of a curvature-depending functional in visual reconstruction. Commun. Pure Appl. Math. 59 (2006) 71-121.

[6] X. Cabré and J. Terra, Saddle-shaped solutions of bistable diffusion equations in all of $\mathbb{R}^{2 m}$. J. Eur. Math. Soc. 43 (2009) 819-943.

[7] G. Dal Maso, An introduction to $\Gamma$-convergence, vol. 8, Progress in Nonlinear Differential Equations and their Applications. Birkhäuser, Boston, MA (1993).

[8] H. Dang, P. Fife and L. Peletier, Saddle solutions of the bistable diffusion equation. Z. Angew. Math. Phys. 43 (1992) 984-998.

[9] E. De Giorgi, Some remarks on $\Gamma$-convergence and least squares method, in Composite media and homogenization theory (Trieste, 1990), MA. Progr. Nonlinear Differ. Eq. Appl. 5 (1991) 135-142.

[10] P. Dondl, L. Mugnai and M. Röger, Confined elastic curves. SIAM J. Appl. Math. 71 (2011) 2205-2226.

[11] Q. Du, C. Liu, R. Ryham and X. Wang, A phase field formulation of the Willmore problem. Nonlinearity 18 (2005) $1249-1267$.

[12] Q. Du, C. Liu and X. Wang, A phase field approach in the numerical study of the elastic bending energy for vesicle membranes. J. Comput. Phys. 198 (2004) 450-468.

[13] J. Hutchinson, $C^{1, \alpha}$-multiple function regularity and tangent cone behavior for varifolds with second fundamental form in $L^{p}$, in Geometric measure theory and the calculus of variations (Arcata, Calif., 1984). Proc. Sympos. Pure Math. Amer. Math. Soc. 44 (1984) 281-306.

[14] J. Hutchinson, Second fundamental form for varifolds and the existence of surfaces minimising curvature. Indiana Univ. Math. J. 35 (1986) 281-306.

[15] J.S. Lowengrub, A. Rätz and A. Voigt, Phase-field modeling of the dynamics of multicomponent vesicles: spinodal decomposition, coarsening, budding, and fission. Phys. Rev. E $\mathbf{7 9}$ (2009) 82C99-92C10.

[16] L. Modica and S. Mortola, Un esempio di $\Gamma^{-}$-convergenza. Boll. Un. Mat. Ital. B 14 (1977) $285-299$.

[17] Y. Nagase and Y. Tonegawa, A singular perturbation problem with integral curvature bound. Hiroshima Math. Journal 37 (2007) 455-489.

[18] M. Röger and R. Schätzle. On a modified conjecture of De Giorgi. Math. Z. 254 (2006) 675-714.

[19] L. Simon, Proceedings of the Centre for Mathematical Analysis, Australian National University. Centre for Math. Anal., Lectures on Geometric Measure Theory, vol. 3. Australian National Univ., Canberra (1984). 